\title{
RESUMEN
}

La Instrucción Pastoral se ocupa predominantemente de principios y directrices pastorales generales, pero hace también aserciones de importancia para el trabajo publicístico en los paises en desarrollo y de misión. Señala la importancia de la comunicación social en el proceso de desarrollo y evangelización. Destaca la responsabilidad de la Iglesia precisamente en este terreno y urge la formación de los correspondientes especialistas, así como el fomento de la investigación y la enseñanza en esta materia. Hace referencia asimismo del papel de los diversos medios de comunicacion (prensa, radio, cine, teatro) y acentúa la necesidad de estructuras adecuadas y de colaboración con otras iglesias y organizaciones. Urge de modo explícito la financiación, en paises en desarrollo y de misión, de proyectos sobre medios de comunicación social. En el futuro no puede desestimarse la comunicación por satélite y una más intensa participación de los jóvenes en los quehaceres propios de la comunicación social.

\section{Der Film und seine Bedeutung für Indien}

\author{
von Gaston Roberge
}

Das Fernsehen spielt in Indien nur eine bescheidene Rolle: im Bereich von Neu Delhi, dem einzigen TV-Zentrum des Subkontinents, gibt es 10.000 Fernsehgeräte. Aber das Fernsehen nimmt in den Plänen, Hoffnungen und Träumen des indischen Volkes einen bedeutenden Platz ein: es wird bald jedes der 500.000 indischen Dörfer erreichen, von denen nur ein Zehntel elektrifiziert ist. Das Fernsehen wird Wunder wirken! Amerikanern, Europäern oder Japanern, die täglich vielleicht drei bis fünf Stunden vor dem Fernseher verbringen, fällt es schwer, die besondere Situation Indiens zu verstehen. Andererseits ist es für die Inder schwierig, die kulturellen Konsequenzen einer möglichen Verwirklichung ihres Fernsehtraums zu begreifen. Was sowohl Inder wie Ausländer allzu leicht übersehen, ist die überragende Bedeutung des Films in diesem Land.

„Es ist erschreckend“, schrieb der Filmkritiker und Filmmacher Chidananda Das Gupta, „daran zu denken, das das einzige Medium, das eine wirkliche Kommunikation im indischen Volk zustande gebracht hat, das kommerzielle Kino ist." Hier liegt die Bedeutung des Films in Indien. Die verblüffende Tatsache, daß Indiens Filmindustrie eine der größten der Welt ist - wahrscheinlich nur von Japan übertroffen -, muß im Zusammenhang eines Landes gesehen werden, das mehr als 500 Millionen Einwohner hat, die mehrere Hundert verschiedene Sprachen und

Gaston Roberge SJ MA ist nach Filmstudien in Los Angeles und praktischen Erfahrungen in Indien jetzt Leiter des "Social Communication Service“ für Nordost-Indien in Kalkutta. 
Dialekte sprechen. Was bedeuten für sie 325 Filme pro Jahr? Trotz seiner gewaltigen Industrie hat Indien noch immer einen unterentwickelten Film und erreicht bei weitem nicht jene zwei Kinoplätze auf hundert Personen, die von der UNESCO als Minimum empfohlen werden. Gleichwohl bleibt das Kino die populärste Massenunterhaltung in Indien. In einer Sprache, die die Massen verstehen und lieben, dramatisiert der Film fortwährend den Verfall eines Volkes, das, von den Errungenschaften der Technologie fasziniert, durch Jahrtausende alte Traditionen gebunden ist.

Das indische Fernsehen scheint die Charakteristika des indischen Rundfunks und Kinos zu übernehmen. Trotz ihrer großen Erfolge werden beide Medien verdientermaßen kritisiert. In ihrer Funktion, das Volk dieses Landes zu erziehen, zu informieren und $\mathrm{zu}$ unterhalten, haben sie weithin versagt. Das Erziehungssystem, eine weitere einflußreiche, reaktionäre Macht in der indischen Gesellschaft, hat zu diesen beiden wichtigen Massenmedien, Rundfunk und Film, keine Beziehung. Das Ergebnis: während eine privilegierte Minderheit ihre Zeit in Schulen und Colleges vergeudet, werden die Massen durch das Kino betäubt. Unter solchen Umständen ist es dringend erforderlich, ein Bewußtsein für die Bedeutung zu entwickeln, die Film und Rundfunk bei der Entwicklung der indischen Nation spielen können. Es besteht die Hoffnung, daß durch die Schaffung einer positiven Einstellung zu diesen beiden Medien auch dem Fernsehen gegenüber eine günstigere Atmosphäre entstehen kann. Dieser Beitrag behandelt allerdings nur den Film.

\section{Der indische Film}

Der Film füllt in Indien ein Vakuum, das durch den Verfall der traditionellen Unterhaltungskünste entstand, als Folge jener kolonialen Praxis, die der landbesitzenden Oberschicht ihre Machtmittel raubte und dadurch die reichen Mäzene der Kunst entfremdete. Die traditionellen Formen der Kunst verloren ihre Beweglichkeit und konzentrierten sich in den Gegenden ihres Ursprungs oder in exklusiven Liebhaberzirkeln. Die Kunstformen, die traditionellerweise vom Landadel unterstützt wurden, hatten ein Massenpublikum, das für die gebotene Unterhaltung nicht zu bezahlen brauchte. Die Tatsache, daß kein anderes Massenunterhaltungsmittel so einfach zu transportieren war, in einem Land, wo Kommunikation noch immer ein Problem ist, machte es dem Film leicht, Fuß zu fassen. Wie der Film allgemein Anleihen bei anderen Kunstformen macht, so übernahm auch die indische Filmproduktion Elemente der folkloristischen und klassischen Theatertradition: Vorrang der Musik, Fehlen einer streng gebauten Fabel, Abschweifungen, große Länge der Veranstaltung. Alle diese Faktoren bestimmen den Charakter des indischen Films: Länge, lose Synthese verschiedener Künste, Fehlen strikt formaler Muster.

Man sollte nicht so vom indischen Film sprechen, als ob es nur eine einzige Art gäbe. Die Künste sind in diesem Land vielgestaltig: es gibt wenigstens zwei Hauptrichtungen der Architektur, zwei musikalische Systeme, vier große Tanzformen. Wie kann man da ein einziges indisches Kino erwarten? Tatsächlich hat der Film in den einzelnen Gebieten Indiens sich in verschiedener Weise entwickelt. Satyajit Rays Filme etwa, die im Westen am meisten bekannt sind, können als ein typisch bengalisches Phänomen bezeichnet werden. Um immer mehr Zuschauer zu erreichen ein dem Kino wesentliches Element - , ist in Indien eine besondere Art von Filmen entstanden, der All-India-Film, der für das sehr differenzierte Publikum dieses Lan- 
des einen gemeinsamen Nenner sucht. Dieser "gesamtindische" Film gebraucht eine Form des Hindi, jener indischen Sprache, die von den meisten gesprochen oder wenigstens verstanden wird; er ist von allen regionalen Besonderheiten gereinigt, also eine Abstraktion. Selbst die Sprache, die er benutzt, wird in dieser Form nirgendwo in Indien gesprochen, ausgenommen vielleicht in den Studios in Bombay, wo sie herkommt.

Seit Entstehen seiner Filmindustrie im Jahre 1912 hat Indien über 10.000 Filme produziert. Rund 1.000 Filme wurden in der Stummfilmzeit gedreht. Zwischen 1931 und 1967 wurden in Indien annähernd 9.000 Filme in den folgenden Sprachen hergestellt:

$\begin{array}{lrlr}\text { Hindi } & 3.908 & \text { Malayalam } & 234 \\ \text { Tamil } & 1.233 & \text { Gujarati } & 129 \\ \text { Bengali } & 1.110 & \text { Punjabi } & 88 \\ \text { Telegu } & 828 & \text { Assamese } & 37 \\ \text { Marathi } & 440 & \text { Oriya } & 36 \\ \text { Kannada } & 254 & & \end{array}$

Seit 1967 hat der Hindi-Film als der "gesamtindische" seine Vorherrschaft noch weiter ausgebaut. Während der Hindi-Film im Norden beliebter ist, genießt der Tamil-Film im Süden größere Sympathien. Die bengalische Filmindustrie ist in den beiden letzten Jahren fast ganz zusammengebrochen.

\section{Der All-India-Film}

Ein erstaunliches Phänomen des All-India-Films ist die Tatsache, daß er sich nicht, wie sein Gegenstück aus Hollywood, in verschiedenen Gattungen entwickelt hat. Der "gesamtindische" Film ist eine Gattung für sich. Er scheint es auch noch einige Jahre zu bleiben, wenngleich eine "Neue Welle“ sich ankündigt; denn Veränderungen geschehen in diesem Land nur sehr langsam.

Die Besonderheiten des All-India-Films können wie folgt beschrieben werden:

a) Überragende Bedeutung des Stars. Häufig werden Künstler für sechs bis zehn Filme engagiert, die zur gleichen Zeit gedreht werden. Die Stars beziehen märchenhafte Gagen und haben einen sehr großen Einfluß auf die Massen, die zu Heldenverehrungen neigen und sich mit keiner lebenden Führergestalt identifizieren können.

b) Koexistenz verschiedener Kunstformen innerhalb des Films, ohne den Versuch, sie $\mathrm{zu}$ einer Einheit $\mathrm{zu}$ integrieren. Wie das Fernsehen von den Werbespots, so wird auch der Film häufig unterbrochen: durch ein Lied oder einen Tanz. Ein „gesamtindischer" Film muß etwa sechs Lieder und drei Tänze haben. Die Schauspieler und Tänzer singen niemals selbst. Die Lieder werden - off screen - von anderen Stars vorgetragen.

c) Die Inhalte sind einfach, denn sie müssen von den einfachen Massen leicht begriffen werden, die of nicht einmal die im Film gebrauchte Sprache verstehen. Zudem schließt die lockere Form des Films, der sich über drei Stunden erstreckt und von Gesang und Tanz unterbrochen wird, eine straffe Fabel aus. Da es für gewöhnlich keine Handlung im aristotelischen Sinn gibt, entfällt die Notwendigkeit einer stren- 
gen Handlungsführung. Die Geschichte kann irgendwo aufhören, denn sie ist nur ein Vorwand, ein Vehikel für Lieder, Tänze, für komische und tragische Situationen.

d) Die Personen im Film haben keine Beziehung zum heutigen Indien. Sie können sehr reich sein oder es über Nacht werden. Sie arbeiten selten. Die Farblosigkeit des indischen Films ist einerseits bedingt durch eine gewisse Neigung zur Verdrängung, aber auch durch die außerordentlich strenge Kontrolle von offizieller oder anderer Seite. Tatsächlich kann kein Film aktuelle Probleme behandeln.

e) Prüderie. Die indische Zensurbehörde ist eine der unbeweglichsten in der Welt. Kußzenen etwa sind auf der Leinwand nicht möglich. Das ist unrealistisch. Wegen dieser wirklichkeitsfernen Zensur in Sachen Sex haben die Filmemacher eine erstaunliche Geschicklichkeit entwickelt, Sexfilme zu produzieren, die - bei einem Minimum an Handhabung - ein Maximum an Anspielung enthalten. Es ist möglich, daß das Publikum in dieser Frage innerlich dem Zensor zustimmt. Die Filmbesucher ziehen vermutlich die länger dauernde Freude an Anspielungen der kurzen Ersatzbefriedigung gezeigter Aktionen vor und lieben die Erotik mehr als den einfachen Sex. Auch in anderen Lebensbereichen respektiert der indische Film die Tabus der indischen Gesellschaft. Figuren, die diese heiligen Bande zu zerbrechen wagen, werden bestraft, die Tradition bleibt geschützt.

f) Die bedeutende Rolle der Frau. Sie opfert sich für das Wohl aller. Sie ist die Hüterin von Tradition und Frömmigkeit, sie hält die Gesellschaft zusammen. Obwohl strukturell ein Patriarchat, ist Indien gefühlsmäßig ein Matriarchat.

Satyajit Ray schrieb über den All-India-Film: „Die Elemente des durchschnittlichen Hindi-Films sind bekannt: Farbe (vor allem Eastman); Lieder (sechs oder sieben) von Stimmen, die man kennt und denen man vertraut; Tänze - Solo oder Gruppen - je verrückter, desto besser; schlechtes Mädchen - gutes Mädchen, schlechter Kerl guter Kerl; Romanze (aber keine Küsse); Tränen, Gelächter, Kämpfe, Verfolgungsjagden; Melodrama; Charaktere, die in einem sozialen Vakuum leben; Wohnungen, die es außerhalb der Studios nicht gibt; Außenszenen in Kulu, Manali, Oty, Kashmir, London, Paris, Hongkong, Tokio. Sehen Sie sich drei Hindi-Filme an, und Sie werden die genannten Ingredienzen finden.“ ("An Indian New Wave?“, in: „Filmfare" vom 8. Oktober 1971).

Das ist die Art von Filmen, die sich die Leute gern ansehen. Selbst viele von denen, die sie überheblich ablehnen, gehören zu den regelmäßigen Besuchern, als ob sie damit zeigen wollten, daß sie wirklich nicht so schlecht sind, wie immer behauptet wird. John M. Culkin, früherer Direktor des "Center for Communications" der Fordham Universität, erzeugte Verwunderung, als er amerikanischen Erziehern sagte, daß die Schüler in Indien mehr Zeit vor Kinoleinwand und Fernsehschirm verbringen als im Klassenzimmer. Culkin hatte eine sehr einfache Rechnung aufgestellt, für die sich vor ihm noch niemand die Mühe genommen hatte. In Indien kann jeder, der will, eine noch erstaunlichere Tatsache feststellen: hier gibt es mehr Menschen, die AllIndia-Filme sehen, als solche, die eine Schule besuchen. Daß das kommerzielle Kinc nicht den Ehrgeiz habe, sein Publikum zu erziehen, ist ein schwacher Trost, wenn man bedenkt, daß es dabei um hunderte Millionen Menschen geht.

Wegen der Beliebtheit des All-India-Films bei den Massen haben die regionalen Filmindustrien Schwierigkeiten zu überleben. Doch nur der regionale Film kann sich wirklich mit aktuellen Problemen auseinandersetzen. 


\section{Dokumentarfilm}

Irgendwann in den vierziger Jahren wurde entschieden, daß bei jeder Kinovorstellung 20 Minuten Erziehungsfilm zu zeigen sei. Diese Forderung entsprang der Sorge um das Publikum, aber auch der Uberlegung, hierdurch künftigen Kurzfilmmachern einen Markt zu eröffnen. Doch es wurden nicht genügend Kurzfilme hergestellt. Deshalb übernahm die indische Regierung selbst die Produktion der notwendigen Kurzfilme und hat folglich den Markt besetzt, der eigentlich für die unabhängigen Kurzfilmproduzenten vorgesehen war.

Die Vorführung von Kurzfilmen schlägt in den Bruttoeinnahmen der Kinohäuser mit $1 \%$ zu Buche. Doch dieses Geld, eine ansehnliche Summe, wird nicht in die Arbeit der Films Division, einer Abteilung des Ministeriums für Information und Rundfunk, die den Film produziert hat, rückinvestiert. Das Budget der Films Division ist fest, unabhängig vom Erfolg der Filme. Films Division ist eine gewaltige Organisation, die - einzig in der Welt - Kurzfilme in 14 Sprachen herstellt, dazu Wochenschauen für mehr als 5.000 Kinos. Jedes Jahr produziert diese Abteilung etwa 40.000 Filmeinheiten. In der Vergangenheit haben die Filme der Films Division allzu deutlich sich in den Dienst der Regierung gestellt. Das ist in der letzten Zeit sichtbar anders geworden. Films Division produziert jetzt auch kritische Filme. Man sollte jedoch nicht übersehen, daß in den Medienprogrammen der Regierung, die für die gesamte Nation von Bedeutung sind, der Film eine wichtige Aufgabe erfüllt. Die Menschen müssen über die Leistungen der Regierung und deren Zukunftspläne informiert werden.

Wenn auch in letzter Zeit eine Reihe von Filmen der Films Division erfolgreich gewesen sind, lassen die indischen Dokumentarfilme das Publikum im allgemeinen unbeteiligt. Die Situation, in der diese Filme gezeigt werden, wird weder von den Filmkritikern, noch auch - leider - von den Filmmachern genügend bedacht. Eine Sache ist es, einen Film zu machen, der internationale Preise einheimst, und eine andere, einem uninteressierten Publikum, das echte Unterhaltung sucht, eine Botschaft zu vermitteln. Dies erklärt das Scheitern der ansonsten gut gemachten Filme, die im Unterricht, aber nicht in Kinosälen verwendet werden sollten.

Die Films Division hat ein Publikum zur Verfügung, das sie bei jeder Kinovorstellung 20 Minuten lang ansprechen kann. Es könnte viel getan werden, um die Kommunikation mit diesem Publikum zu verbessern. Es ist symptomatisch, daß die Pädagogen dieser Möglichkeit, die Massen zu erreichen, keine Bedeutung beimessen.

\section{Eine Neue Welle in Indien?}

Viele Filmmacher sind unzufrieden mit den Bedingungen, die ihnen von der Filmindustrie auferlegt werden. Durch erweiterte Erziehungsarbeit, durch die Kenntnis ausländischer Filme in Filmgesellschaften und die Präsenz des amerikanischen Films in allen großen Städten ist eine wachsende Zahl von Menschen mit dem indischen Film immer weniger zufrieden. Diesem Publikum und einer Gruppe politisch bewußter Leute möchten sich einige Filmmacher zuwenden, deren anerkannter Führer der Bengale Mrinal Sen ist. Sein erster Hindi-Film, „Bhuvan Shome“, den man als Bengali-Film in Hindi-Sprache bezeichnen kann, gewann 1970 den indischen Staatspreis. Der Film wird von indischen Kritikern als außergewöhnlich beurteilt. Durch Unterstützung der indischen Regierung, die neue Versuche von jungen und alten 
Filmmachern durch Anleihen bei der Film Finance Corporation subventioniert, entsteht ein neuer Typus von Filmen, die experimentell oder zumindest anders sein wollen. Satyajit Ray warnte in einem Artikel, der in der populären Zeitschrift "Filmfare" erschien, die begeisterten Experimentierer vor einer leichtfertigen Nachahmung der "Neuen Welle" westlicher Prägung. Er betonte mit Nachdruck, daß die Filmmacher versuchen sollten, ein möglichst großes Publikum zu erreichen, anstatt sich auf bestimmte Kreise zu beschränken.

Das Experimentalkino in Indien, wenn es jemals entstehen sollte, und es sieht nicht danach aus, wird vermutlich sofort politisches Kino werden. Das ist höchst wünschenswert. Es ist bezeichnend, daß es in diesem Land keinen Underground-Film zu geben scheint. Gelegentlich werden blue movies (Pornofilme) in $8 \mathrm{~mm}$ gezeigt, aber es gibt keine Underground-Film-Produktion, die den Menschen helfen könnte, Probleme von allgemeinem Interesse zu bewältigen. Der erste Schritt zu einem Underground-Kino ist die Unterstützung vonseiten der Regierung durch die Film Finance Corporation, die kaum als Untergrund-Organisation bezeichnet werden dürfte.

\section{Indische Filme im Ausland}

Die meisten Menschen außerhalb Indiens, sofern sie überhaupt eine Vorstellung vom indischen Film haben, werden ihn mit den Werken von Satyajit Ray identifizieren, ebenso wie das japanische Kino mit den Filmen von Akira Kurosawa gleichgesetzt wird. Die Filme Rays bilden eine eigene Kategorie. Er verdient die Ehrungen, die er erfahren hat, seit sein "Pather Panchali" 1955 in Cannes anerkannt wurde. Seitdem hat Ray fast 20 Filme verschiedener Art fertiggestellt. Das kürzlich erschienene Buch von Marie Seton, der Biografin Sergei Eisensteins, mit dem Titel „Portrait of a director, Satyajit Ray" (Dennis Dobsch, London, 1971), ist ein unentbehrlicher Begleiter für Ray-Filme außerhalb Indiens. Seine Werke werden auf diese Weise in Europa und Amerika leichter zugänglich sein, Marie Setons Buch wird zu einem besseren Verständnis verhelfen. Andererseits wird es aber auch dazu beitragen, Satyajit Ray als den indischen Regisseur weiter zu isolieren.

Indien hat seit den Tagen von Dada Saheb Phalke (1870-1944), seinem ersten bedeutenden Regisseurs, wiederholt versucht, seine Filme zu exportieren. Bisher sind die Hauptabsatzgebiete der Ferne und Nahe Osten, Afrika, Burma, Ceylon und der Persische Golf. Export indischer Filme in den Westen gibt es praktisch nicht. Rußland, der wichtigste Importeur, hat zwischen 1949 und 1963 von Indien 26 Filme übernommen. In Großbritannien, das Indien von allen westlichen Ländern am besten kennt, wurden seit 1945 zwölf indische Filme gezcigt. In den USA sind praktisch nur einige Filme von Ray etwas bekannt. Tatsächlich sind die meisten indischen Filme, die von Ray ausgenommen, für das westliche Publikum ungeeignet. Zudem zeigt man bei der Auswahl für internationale Filmfestivals häufig keine glückliche Hand. So bleiben indische Filme fast gänzlich unbekannt, und auch über die Bedeutung des Kinos im Leben der indischen Nation weiß man im Westen wenig. Doch ist dies nur der Teilaspekt eines umfassenderen Phänomens, nämlich der totalen Unkenntnis des Westens gegenüber Indien, das heute fast $20 \%$ der Weltbevölkerung ausmacht. So bleibt es Indien selbst überlassen, sich bekannt zu machen. Aber sollten nicht auch die westlichen Länder sich bemühen, dieses Land mit seiner vielfältigen Kultur kennen und schätzen zu lernen? 


\section{Filmerziebung}

Auf verschiedenen Ebenen werden Versuche unternommen, die Filmerziehung in Indien auszubauen. Diese Bemühungen bleiben allerdings durch finanzielle Engpässe begrenzt, und sie finden - im Ausland wie in Indien - wenig Unterstïtzung.

Regierungstätigkeit: Das Film and Television Institute of India wurde 1961 gegründet. Es verleiht Diplome für alle Berufe der Filmbranche und zählt 150 Studenten. Fernsehkurse bestehen bisher noch nicht. Von Anfang an war das Institut, vor allem durch seine professionellen Studios, gut ausgerüstet. In den zehn Jahren seines Bestehens hat es bedeutende Schauspieler und Regisseure hervorgebracht. Das nationale Film-Archiv wurde ebenfalls in den frühen sechziger Jahren errichtet. Es hat eine Sammlung von 250 indischen und etwa 50 ausländischen Filmen. Durch den Verleih einiger seiner Filme spielt es heute schon eine wichtige Rolle. Die Film Finance Corporation wurde eingerichtet, um den Filmmachern finanziell $\mathrm{zu}$ helfen. Einige als Neue-Welle-Filme bezeichnete Werke wurden mit seiner Unterstützung produziert. Die Filmzensur in Indien untersteht dem Minister für Information und Rundfunk. Eine Kommission hat, auf Grund einer kürzlich durchgeführten Untersuchung, die sich mit der Zensur befaßte, wertvolle Vorschläge erarbeitet.

Filmclubs: Die Federation of Film Societies hat in Indien etwa hundert Mitgliederclubs mit einer Gesamtmitgliederzahl von rund 80.000. Allein in Calcutta gibt es 14 Clubs, von denen manche bis zu 2.000 Mitgliedern zählen. Viele Clubs haben ihre eigenen Zeitschriften. Aber das Leben der Clubs wie das ihrer Zeitschriften ist oft nur von kurzer Dauer. Der trotz der Bemühungen der Federation bestehende Mangel an Koordination zerstört jede Planung und verhindert, daß eine wirksame Bewegung entsteht. Es gibt verhältnismäßig wenig Filme, und ihre Verbreitung ist schwierig. Die Hilfe der Regierung ist begrenzt. Wenngleich die Clubs durch Verbreitung von Filmen sich beim Publikum verdient gemacht haben, konnten sie die Rolle, die sie hätten spielen müssen, nicht ausfüllen. Sie tun selten mehr, als Filme zu zeigen, und sind unfähig, auf Dauer ein ernsthaftes Filmstudium zu organisieren. Vielleicht haben sie sogar noch dazu beigetragen, die Kluft zu vertiefen zwischen einem Fachpublikum, das die indischen Filme nicht sieht, und dem Massenpublikum, das sich an All-India-Filmen erfreut.

Andere Bemühungen: Um die Ziele der Filmclubs zu unterstützen, engagierte die indische Regierung in der Mitte der fünfziger Jahre Marie Seton für Vorträge über Film überall im Lande. „Film Appreciation“, eine Broschüre von Marie Seton, wurde von einer Regierungsorganisation publiziert. Doch erst gegen Ende der sechziger Jahre organisierte man diese Kurse so, daß auch ein breiteres Publikum von der Filmerziehung erreicht werden konnte. Eine frühere Initiative des Film Institute war im Jahre 1967 ein Vierwochenkurs für 50 Leute, darunter einige von Films Division, den Prof. Satish Bahdur angeregt und geleitet hatte. Einige Erziehungsinstitutionen haben gelegentlich Filmseminare veranstaltet. Für den Gebrauch an Hochschulen wurde ein Buch veröffentlicht. Um die Arbeit der Filmgesellschaften und der Filmpädagogik an den Schulen zu unterstützen, wäre unbedingt eine Organisation erforderlich, die wie das British Film Institute alle Arten von Publikationsmöglichkeiten, Seminaren, klassischen Filmauszügen usw. anbietet.

Pädagogische Filme werden in großer Zahl von Schulen verwendet. In Indien sind etwa 7.000 solcher Filme von ausländischen Missionen und verschiedenen Regierungs- 
stellen verfügbar. Aber diese Filme sind nicht bekannt, und die Lehrer werden zu ihrem Gebrauch nicht angeleitet. Es müßte zunächst ein umfassender Gesamtkatalog aller im Land verfügbaren 16-mm-Filme erstellt werden, mit Anweisungen, wie die Filme im Unterricht verwendet werden können.

\section{Literaturhinweise:}

1. Barnouw, E., und Krishnaswamy, S.: Indian Film, Orient Longmass 1963, XI, 301 Seiten.

2. Das Gupta, Chidananda: Indian Film Today, in: „Film Quarterly“, Bd. 22, Nr. 4, 1969, S. $27-35$.

3. „Four Times Five $/$ The Films Division. Informations- und Rundfunkministerium der Regierung von Indien, 100 Seiten.

4. - Charging the Windmills, in: „Four Times Five ${ }^{\star}$, S. 33-35.

5. Indian Talkie 1931-1956. Silver Jubilee Souvenir. Film Federation of India. Bombay 1956, VII, 216, XXVIII Seiten.

6. Mohan, Jag: Two Decades of the Films Division. Informations- und Rundfunkministerium der Regierung von Indien, 1969, 100 Seiten.

7. Parrain, Philipp: Regards sur le cinema indien. Paris 1969, 400 Seiten.

8. Ray, Satyajit: An Indian New Wave?, in: „Filmfare“, Bombay, 8. Oktober 1971.

9. Report of the Enquiry Committee on Film Censorship. Informations- und Rundfunkministerium der Regierung von Indien, 1969, 202 Seiten.

10. Roberge, Gaston: Mass Communication and Man, Elements of Mass Communications and Film Appreciation for Indian High Schools. Provisional edition. Calcutta 1971, 176 Seiten.

11. Seton, Marie: Film Appreciation. National Institute of Audio-Visual Education, New Delhi (1962?), 70 Seiten.

12. Dieselbe: Portrait of a Director, Satyajit Ray, London 1971, 350 Seiten.

13. Toeplitz, Jerzy: Indian Films and Western Audiences, Paris: UNESCO, 1964. Ubersetzt aus dem Französischen, Bombay: Anandam. Hektographierte Ausgabe, 55 Seiten.

\section{SUMMAR Y}

Television is going to be of greater importance in India, and there are still many unexplored possibilities. But for the time being, film still fulfils a need created by the old methods of entertainment. The all-Indian film, with its artificial Hindi language, is the biggest category. The main topics are entertainment, through stars, songs and dances, simple content without any relation. Documentaries introduced by the Government tend to be attached to every show, but leave the public untouched. The influence of films made in the west is growing. Outside India, the Indian film has almost no impact. Various attempts have been made in the domain of Film Appreciation, but there is still much work to be done.

\section{RESUMEN}

La televisión ocupará próximamente en la India un papel importante y ello implica una serie de imprevistas posibilidades. Pero el cine sigue llenando un vacío, ocupando el lugar del tradicional arte de la conversación. Las películas „para toda India“, dobladas en un artificial idioma hindú, son mayoría y constituyen un género aparte. Predominan las producciones de pasatiempo, salpicadas de estrellas, canciones y bailes, con argumentos sencillos sin conexión alguna con la India actual. El film documental, producido bajo los auspicios del gobierno y plato obligatorio en cada sesión de cine, no llega a interesar al público. Aumenta por otra parte la influencia del cine occidental. En el extranjero el cine indio tiene muy poco peso. En el terreno de la formación cinematográfica se hacen algunos ensayos actualmente. Pero queda todavía mucho por hacer. 